\title{
Evaluación de características asociadas a los egresados IPB - IB de la Universidad Francisco de Paula Santander en el período 200 I a 20 I I con fines de acreditación del programa
}

María Alejandra Tarazona Oliveros ${ }^{1}$, Deisy Milena Espinel Urbina², Sirley Karina Arroyo Solano ${ }^{3}$, Yaneth A. Muñoz Peñaloza ${ }^{4}$

\section{Resumen}

El estudio se enfocó en la Evaluación de características asociadas a los egresados del Programa IPB-IB, donde se elaboró un sistema de información de los mismos en un periodo comprendido de 2001 a 2011, como datos personales, desarrollo laboral y estudios realizados, contando con la colaboración de las diferentes dependencias donde se encuentra esta información, tales como: Secretaria General, oficina de planeación, oficina de egresados, Biblioteca Eduardo Cote Lamus, Programa Académico IPB-IB, Ministerio de Educación Nacional y la Asociación Colombiana de Ingenieros Biotecnológicos. Además, se estableció una comunicación con los egresados mediante vía telefónica y mediante el correo del Programa. Por otra parte, se realizo un análisis acerca de la inserción laboral y puesto de trabajo de los mismos, donde primeramente se creó un Directorio de Egresados, en el cual podemos encontrar el lugar de trabajo donde se encuentra ubicados por sectores; seguidamente se elaboró un cuadro, alli se muestra el puesto de trabajo asignado a cada uno de ellos y el área de desempeño, así como la característica principal de la empresa asociada al desempeño del egresado en la misma. Por otra parte, se creó un formato tipo encuesta dirigido a los egresados, donde se evaluó el impacto del trabajo desarrollado en cada una de las entidades; se elaboró una estrategia de seguimiento tanto para los egresados de la UFPS como para los egresados del Programa IPB-IB. Por último, se creó un Plan Estratégico, en donde se trabajaron las características asociadas a los egresados, un Plan Operativo con las respectivas actividades y tareas a ejecutar y un formato para evaluar el desempeño por parte de los estudiantes en las diversas empresas como trabajo de grado.

\section{Abstract}

The study focused on the evaluation of characteristics associated with graduates IPBIB program, which produced an information system in the same period of 2001-2011, including personal data, job development studies, with collaboration of different departments where this information, such as: Secretary General, planning office, alumni

${ }^{1}$ Estudiante de Ingeniería Biotecnológica, Universidad Francisco de Paula Santander. alejita_123@hotmail.com ${ }^{2}$ Estudiante de Ingeniería Biotecnológica, Universidad Francisco de Paula Santander. espinel_1@ @ hotmail.com ${ }^{3}$ Estudiante de Ingeniería Biotecnológica, Universidad Francisco de Paula Santander. skas 17@ @otmail.com ${ }^{4}$ Directora Ingeniería Biotecnológica, Directora del proyecto, Universidad Francisco de Paula Santander. 


\section{Ecomatemático}

office, Library Eduardo Cote Lamus, IPB -IB Academic Program, Ministry of Education and the Colombian Association of Engineers Biotechnology. In addition, established communication with the graduates by telephone and by mail program. Moreover, an analysis was performed on job placement and job thereof, where first created an Alumni Directory, in which we can find the workplace where it is located by sector, then developed a picture, there is shown the job assigned to each performance and area, as well as the main feature of the company associated with the performance of graduates in the same. On the other hand, created a standard format directed graduates survey, which evaluated the impact of the work done in each of the entities, we developed a monitoring strategy for both graduates UFPS to IPB Program -IB. Finally, we created a Strategic Plan, where you worked the characteristics associated with the graduates, an Operational Plan with the respective activities and tasks to be performed and a format for evaluating performance by students in various companies such as work grade.

Palabras claves: Plan Estratégico, Plan Operativo, Consejo Nacional de Acreditación Impacto, Seguimiento.

\section{Introducción}

$\mathrm{E}$ estudio de egresados resulta de gran importancia, ya que mediante investigación de campo permite conocer la situación actual y el desempeño profesional de sus egresados, sus necesidades y requerimientos, así como evaluar la pertinencia curricular de los programas educativos y sus posibles adecuaciones. Esta información es sumamente útil para orientar la formación de los nuevos profesionales y el diseño de programas académicos que fortalezcan e impulsen estrategias de mejoría continua en el ámbito académico.

Los programas e instituciones pueden aspirar a la acreditación a través de un mejoramiento continuo, alcanzando niveles cada vez más exigentes de calidad, reconocimiento otorgado por el Ministerio de Educación Nacional sobre la alta calidad de un programa o de una institución basándose en un proceso previo de autoevaluación en donde intervienen las comunidades académicas y el Consejo Nacional de Acreditación, CNA. Las universidades reconocen que la acreditación contribuye al mejoramiento de los programas académicos, desarrollando autoevaluación permanente; ejecutando planes de mejoramiento siendo un punto de partida para afianzar la autonomía universitaria.
El presente estudio está enfocado a conocer y evaluar dentro del periodo de 2001 a 2011 para una generación de egresados la eficiencia profesional, la aceptación en el mercado laboral y la correspondencia entre las áreas profesionales y las necesidades laborales.

\section{Materiales y Métodos}

El estudio se realizó en la Universidad Francisco de Paula Santander en el Programa académico Ingeniería Biotecnológica en cuatro etapas. Se inicio con la elaboración de un Sistema de información acerca de los egresados en un periodo de 2001 a 2011 contando con la colaboración de diferentes dependencias de la Institución como la Oficina de egresados, el Programa Académico de IPB-IB, la Biblioteca Eduardo Cote Lamus, entre otras. En segundo lugar, se realizo un análisis tanto del puesto de trabajo como la inserción laboral de estos, mediante la elaboración de un Directorio de Egresados y un cuadro donde se identifica el área de su desempeño y el cargo que ocupa cada uno de ellos. Además, se diseñó un Formato tipo encuesta dirigido a los egresados para determinar el impacto tanto en el medio laboral como académico, el cual consta de 10 preguntas cerradas y concretas. En 


\section{Ecomatemático}

tercer lugar, se creó una estrategia de seguimiento tanto para los egresados de la UFPS como para los del Programa, para su registro y control y así poder establecer una relación permanente con los mismos. Por último, se diseñó un Plan Estratégico, Plan Operativo y Formato de Evaluación de Trabajo de Grado, donde se trabajaron las características asociadas a los egresados contenidas en el CNA.

\section{Resultados y Discusión}

Elaboración de un sistema de información de los egresados del Programa de IPB - IB en el periodo comprendido de 2001 a 2011 de la Universidad Francisco de Paula Santander.

Realizada la recopilación de la información, se procedió a realizar la organización de la misma, ya

Cuadro 1.

\begin{tabular}{|c|c|c|c|}
\hline $\mathrm{N}_{\mathrm{a}}$ & FUENTE & INFORMAGON & REGISTRO \\
\hline 1 & SECRETARIA GENERAL. & $\begin{array}{l}\text { Fecha de grado, documento identidad, } \\
\text { apellidos, nombres, libro, Folio, Acta y } \\
\text { Profesion. }\end{array}$ & Digital \\
\hline 2 & OFICINA DE PLANEACION & $\begin{array}{l}\text { Numero de graduados por ano, cuadros y } \\
\text { graficas (estadisticas). }\end{array}$ & Digital \\
\hline 3 & B.U.OFICINA EGRESADOS & $\begin{array}{l}\text { Directorio de Egresados (digital) } \\
\text { Formatos, encuestas }\end{array}$ & $\begin{array}{l}\text { Fisico } \\
\text { Digital }\end{array}$ \\
\hline 4 & BIBLIOTECA & $\begin{array}{l}\text { Trabajos de Grado, autores (digital, web UFPS: } \\
\text { http://alejandria.ufps,edu,co:81/biblioteca/ } \\
\text { home ufps }\end{array}$ & Digital, web UFPS \\
\hline 5 & $18-1 P B$ & $\begin{array}{l}\text { Cuadro resumen de Trabajo de Grado (digital), } \\
\text { Formatos de Egresados (fisico y digital). } \\
\text { Directorio de Egresados (digital) } \\
\text { Comunicacion virtual: correo electronico de la } \\
\text { Carrera: inghiotechologicaeufoseduce y } \\
\text { GRUPO FACEBOOK INGENIERIA } \\
\text { BIOTECNOLOGICA. }\end{array}$ & $\begin{array}{l}\text { Fisico } \\
\text { Digital }\end{array}$ \\
\hline 6 & MEN & 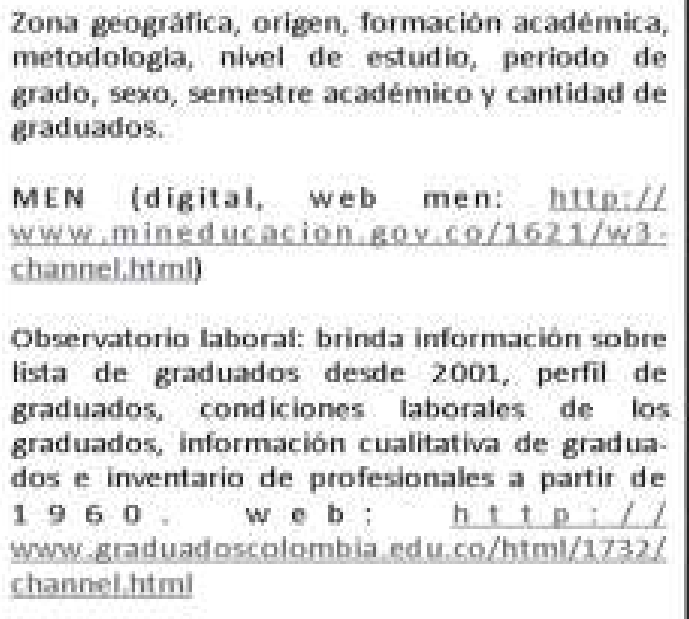 & Digital, web MEN \\
\hline 7 & $\begin{array}{l}\text { ASOCIACION DE } \\
\text { EGRESADOS IB (ACOINBI) }\end{array}$ & $\begin{array}{l}\text { Objeto social, Mision, vision, Politicas, valores } \\
\text { institucionales, portafolio de servicios y socios } \\
\text { fundadores. }\end{array}$ & Digital \\
\hline
\end{tabular}




\section{Ecomatemático}

que esta se encontraba distribuida en diferentes documentos suministrados por las diversas dependencias teniendo en cuenta el orden de importancia de cada una en cuanto a la información que manejan, como se muestra en el cuadro 1.

El sistema de información de los egresados IPB-IB, queda constituido por las fuentes de Información que figuran en el Cuadro 1, en especial la información en el Directorio de Egresados del Programa IPB-IB. El Directorio se debe actualizar de forma periódica (anual) y mantenido la comunicación con los egresados.

Para mejorar la comunicación con los egresados se propone un encuentro institucional de egresados cada dos años.

Además, se elaboro un formato dirigido a los egresados, solicitando la información requerida, el cual se envió a través del correo electrónico de la Carrera, como se muestra en la figura 1.

Figura 1.

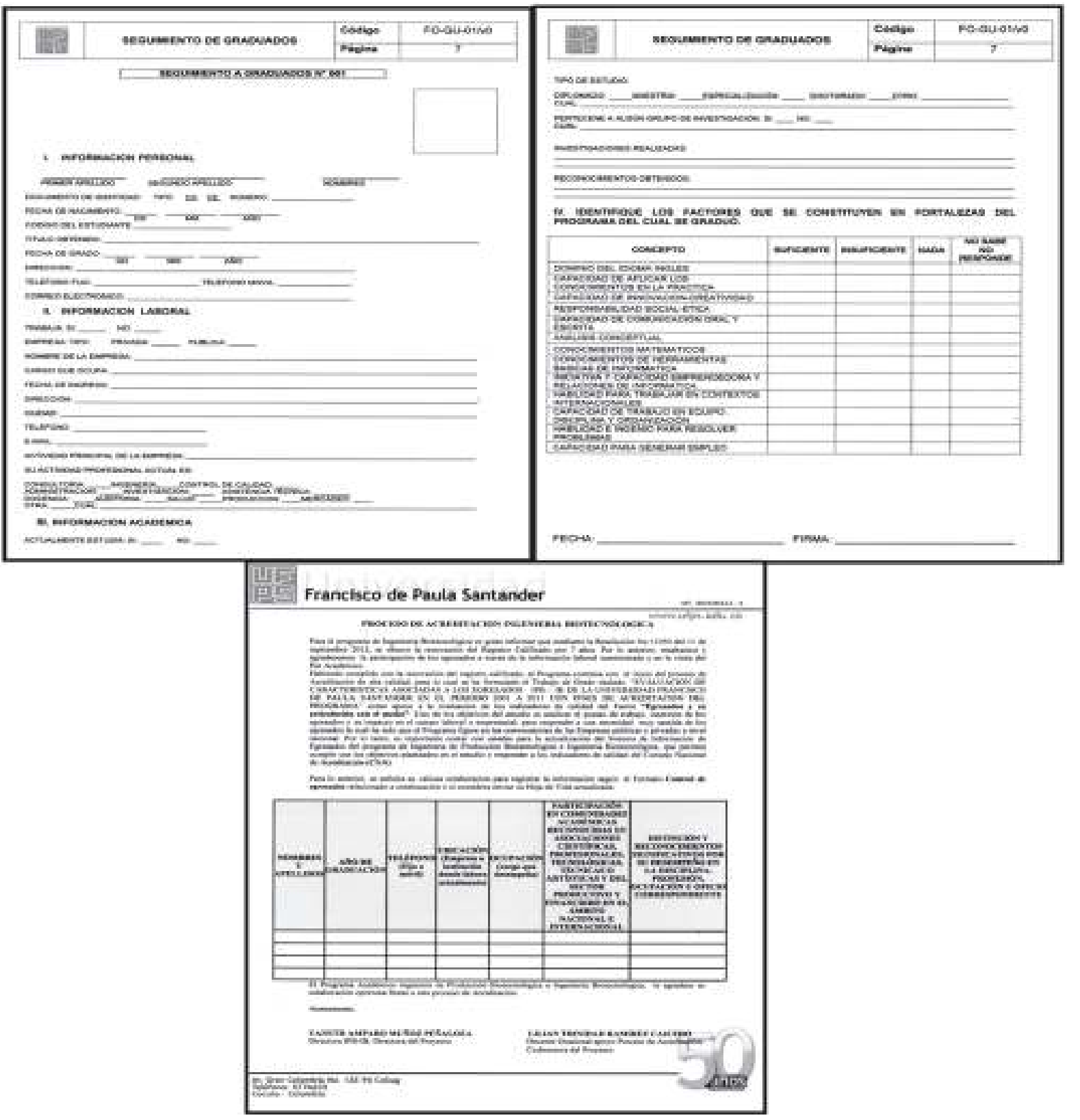




\section{Ecomatemático}

2. Análisis de la inserción Laboral de los Egresados.

Los egresados en el periodo 2011-2011 corresponden a 313 y se trabajó una muestra de 174 egresados (56\%) A los egresados se les solicitó información básica actualizada como: teléfono, ubicación, cargo o desempeño actual y la empresa donde se encuentra laborando.

El análisis del comportamiento de la muestra de los 174 egresados muestra, que el 19\% se encuentran el sector ambiental, el 14\% en el sector industrial, el $12 \%$ en el sector agrícola, el $20 \%$ en el sector educativo y el $20 \%$ independiente (laboran fuera del perfil son 35). Un 10\% de los egresados están realizando estudios de postgrado (no laboran son 17) y el otro $5 \%$ se desempeñan profesionalmente (otros sectores) y simultáneamente realizan estudios de postgrado, como se muestra en la figura 2.

Figura 2. Sectores en los cuales actúan los egresados del programa durante el periodo 2001-2011.

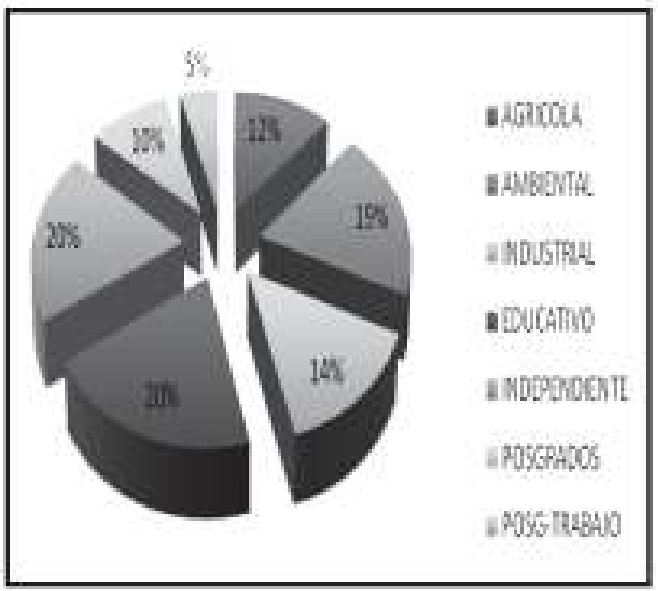

De los 174 egresados, 157 se encuentran laborando como se muestra en el comportamiento del índice de empleo entre el periodo 2001 - 2011, como se muestra en el cuadro 2 y figura 3.

Cuadro 2. Índice de empleo de egresados en el período 2001. 2011.

\begin{tabular}{|l|c|}
\hline Muestra & 174 \\
\hline Laborando & 157 \\
\hline Índice de empleo & 0.90 \\
& \\
\hline
\end{tabular}

Figura 3. Comportamiento del índice de empleo de egresados en el período 2001-2011.

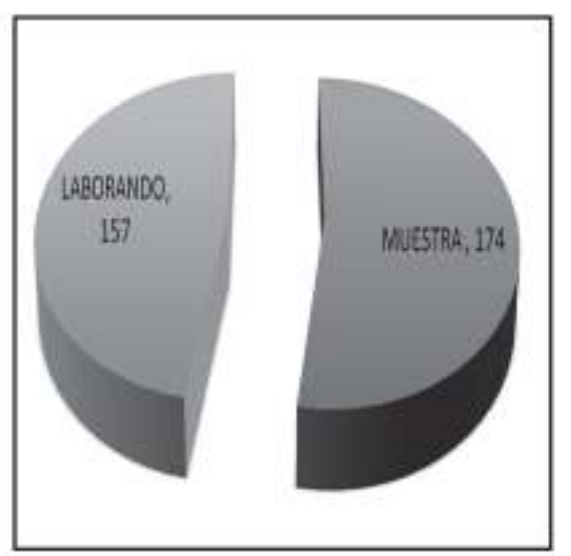

De los 174 egresados, el 75\% se encuentran laborando dentro del perfil. Estos resultados muestran que existe una correlación entre el perfil de formación de los egresados del programa y la ubicación de los mismos en el mercado laboral.

3. Análisis del Puesto de Trabajo de los Egresados.

El potencial de Empresas a nivel local y nacional en las cuales pueden laborar los egresados del Programa, se obtuvo con la actualización y complementación del Directorio de Empresas.

Para delimitar el análisis del puesto de trabajo se evalúo la inserción laboral de los egresados IPBIB por los Departamentos en Colombia, contando con la Información del Directorio actualizado de Egresados, como se observa en la figura 4.

Figura 4. Distribución de la vinculación de los egresados IPBIB por Departamento en Colombia.

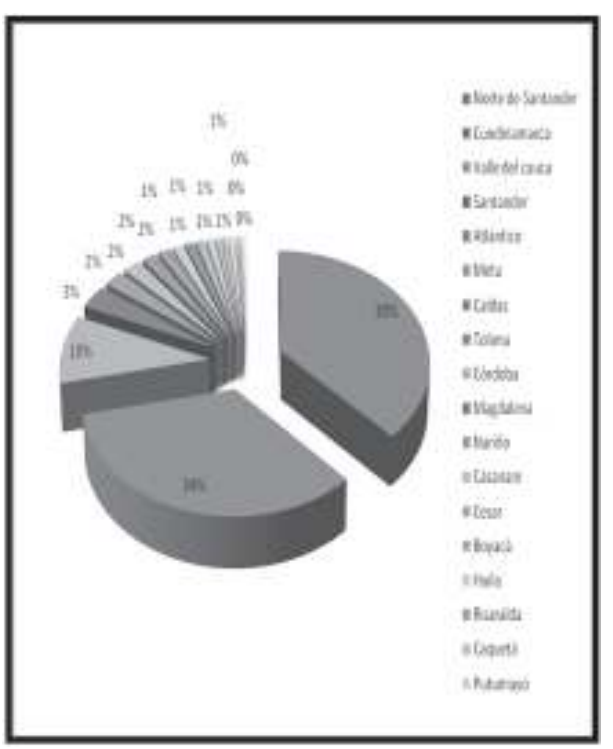




\section{Ecomatemático}

En la figura 4, se aprecia que el Departamento con el mayor porcentaje de inserción de egresados en las empresas o Entidades con un 38\% es el Norte de Santander, seguido del Departamento de Cundinamarca con un $34 \%$ y Valle del Cauca con un $10 \%$, presentando éstos los más altos porcentajes en cuanto a la vinculación de los egresados a las entidades por Departamentos. De acuerdo a los resultados obtenidos se seleccionó el Departamento Norte de Santander para el estudio del puesto de trabajo.

4. Análisis del Impacto de los Egresados.

Para realizar el análisis del impacto de los egresados, se elaboró y envió el Formato tipo encuesta a 174 egresados vía web, como se observa en la figura 5 .

Con estos resultados obtenidos mediante la aplicación del Formato tipo encuesta, podemos obser- var que la totalidad de los egresados a encuestar no respondieron o nos suministraron la información requerida por la misma, ya que solo de 174 de ellos atendieron a esta 64 egresados, siendo esto desfavorable al momento de realizar un análisis cuantitativo y al tabular la información, para así poder mostrar resultados y análisis favorables para el presente estudio y poder contar con la opinión de la totalidad de los egresados del Programa.

5. Estrategia de Seguimiento de los egresados por parte del Programa.

La Oficina de Egresados de la Universidad Francisco de Paula Santander ejecuta un Plan de seguimiento a los egresados surgiendo de la necesidad de mantener una estrecha comunicación entre la U.F.P.S y sus Egresados, a través del registro, control y seguimiento de los mismos, como se observa en la figura 6.

Figura 5.

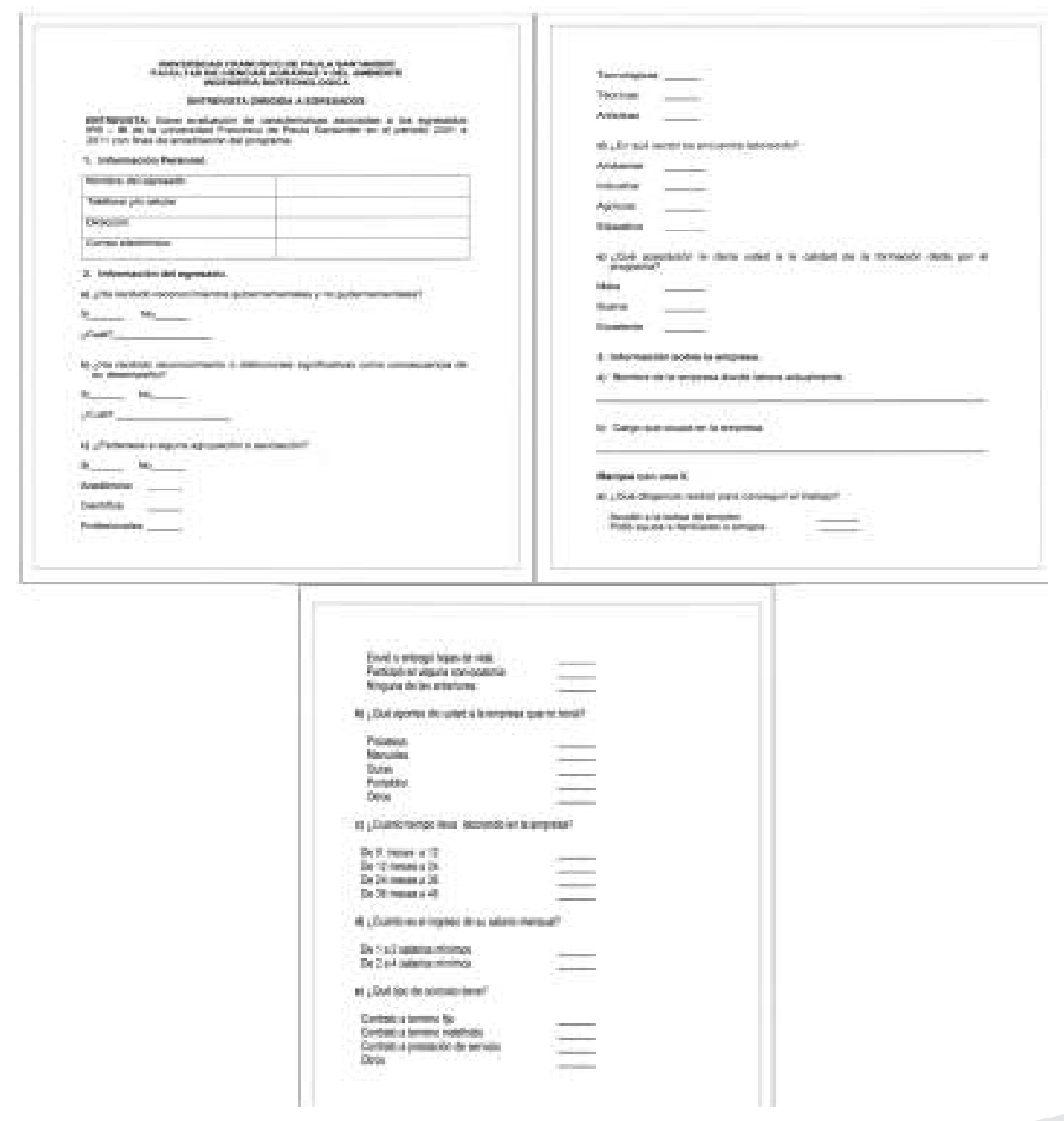




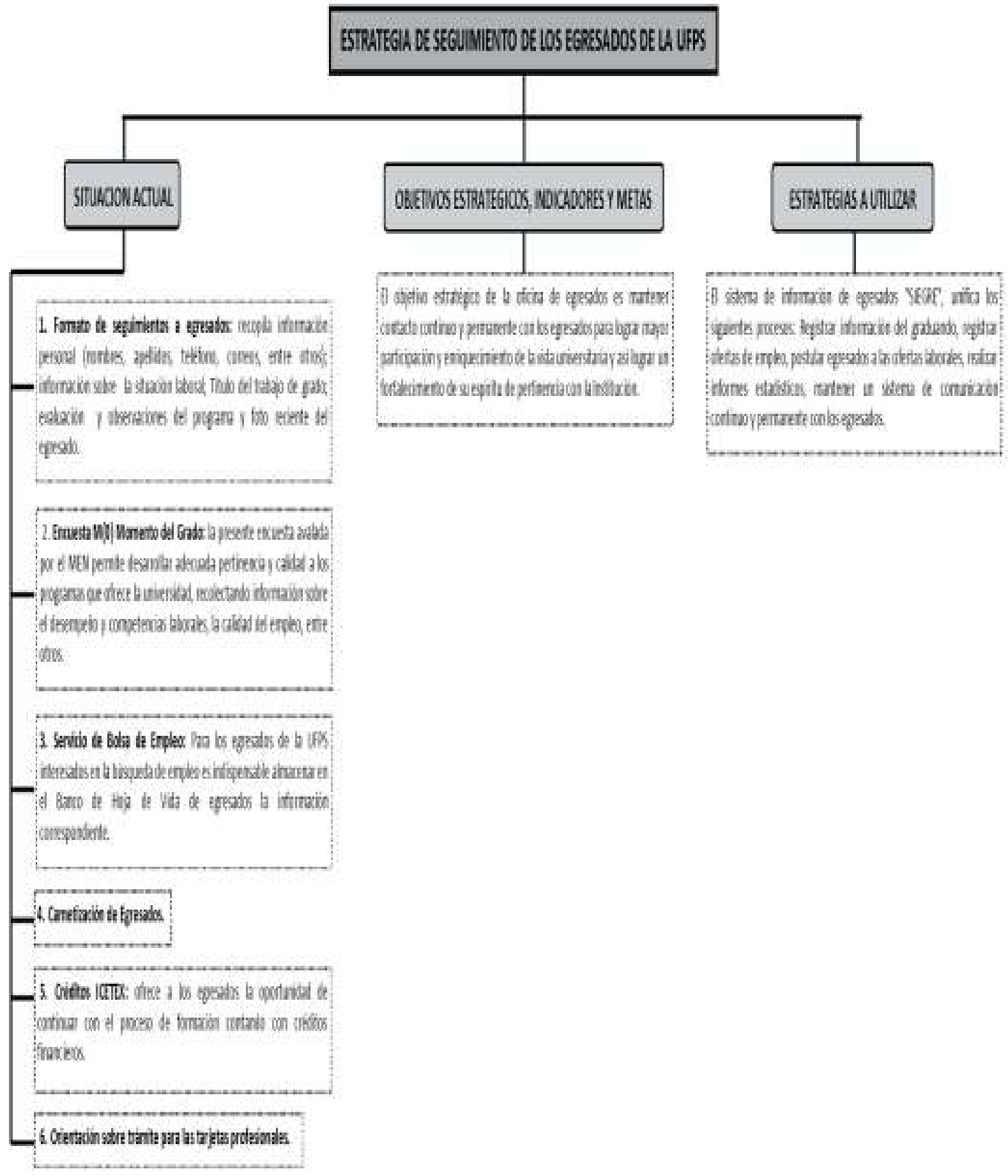

La estrategia de seguimiento para los egresados del programa se realiza por medio del Plan Estratégico de seguimiento de los egresados IPB-IB, el cual hace uso de la información de los egresados
IPB-IB a nivel institucional y del Observatorio Laboral, como se muestra en la figura 7 .

6. Plan Estratégico para trabajar las características asociadas a los egresados. 


\section{Ecomatemático}

Figura 7.

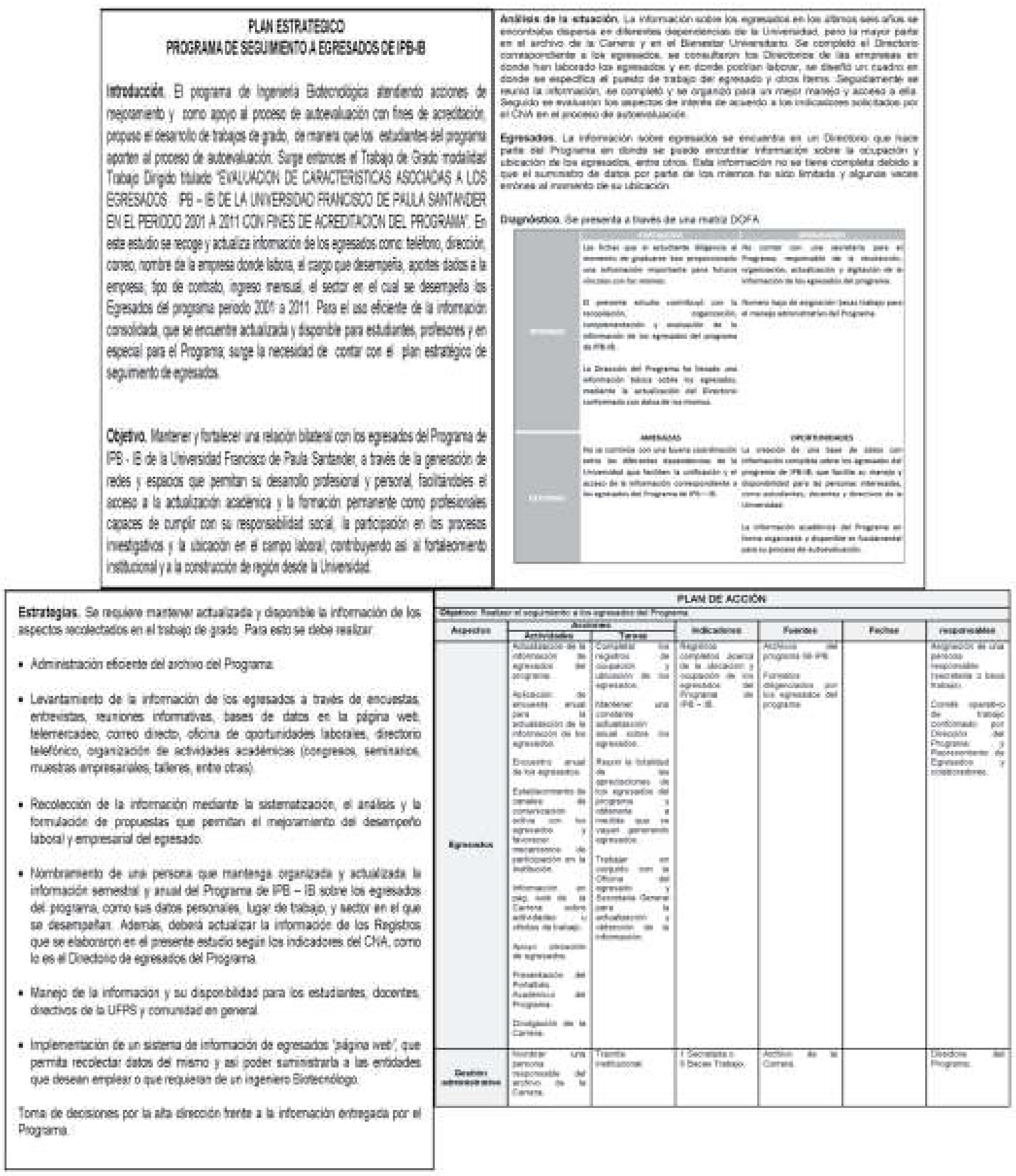

El Plan Estratégico elaborado permite trabajar las características del CNA del FACTOR 9: IMPACTO DE LOS EGRESADOS AL MEDIO, asociadas a los egresados del Plan de estudios de IPB-IB, contempla Plan Operativo, cuyo objetivo principal es promover y fortalecer las acciones entre la universidad y los egresados mediante el análisis de impacto, experiencias y nivel de desempeño para el mejoramiento y la calidad del programa y de esta manera cumplir con las normas establecidas por el Sistema Nacional de Acreditación. También se elaboró un Formato de Evaluación de Trabajo de Grado, para contar con información relacionada a las características del FACTOR 9, como se puede apreciar en la figura 8 . 


\section{Ecomatemático}

Figura 8

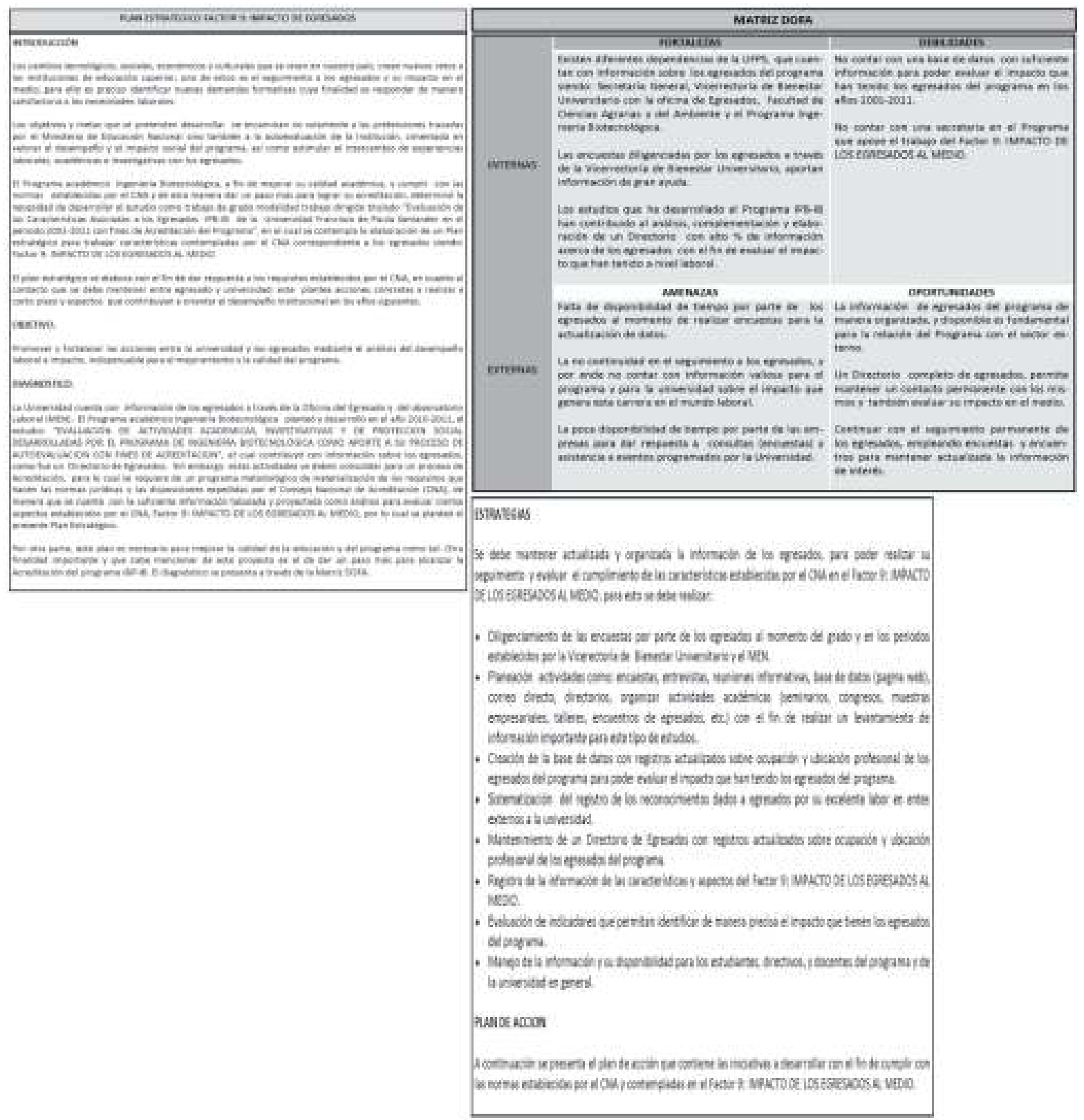

\section{Conclusiones}

El sistema de información de los egresados de IPBIB, corresponde al conjunto de las diferentes fuentes de información de la Universidad representados registros, estadísticas, formatos y Directorios. Siendo el Directorio el que centra toda la información de los Egresados IPB-IB.

Son 313 egresados de IPB-IB, en el periodo 20012011, la muestra para el estudio fue de 174 egresa- dos (56\%) y se determinó un índice laboral de 0.9 La inserción laboral de los egresados IPB-IB de acuerdo a la Empresas se encontró en orden descendente en: sector educativo, ambiental, industrial y agrícola. El Departamento Norte de Santander presentó la mayor inserción laboral de los egresados IPB-IB a nivel nacional, siendo seleccionado para el análisis del puesto de trabajo.

El potencial laboral determinado en el estudio, se dio a conocer mediante comunicación específica 


\section{Ecomatemático}

y el Portafolio Académico del Programa, enviado a las empresas vía web.

Se elaboró un formato tipo encuesta, donde se identifican los aportes realizados por los egresados y los beneficios obtenidos por las empresas. Con este formato, se logró encuestar a 64 egresados entre un total de 174 , con los cuales se obtuvo información sobre el entorno laboral de los mismos y el impacto generado por su desempeño en cada una de las entidades donde han laborado o se encuentran laborando. Con cada una de las preguntas planteadas, se pudo determinar la opinión que tienen los egresados acerca de la calidad del Programa y la generación de empleo que han obtenido con los estudios cursados y su aprendizaje.

Se estructuró y elaboró el Plan estratégico de seguimiento a Egresados de IPB-IB con referencia del marco institucional de la Vicerrectoría de Bienestar Universitario.

El Plan Estratégico fue elaborado con el fin de tener un método concreto para trabajar de manera ordenada las características asociadas a los egresados del Programa IPB-IB, cuyo objeto es coordinar los objetivos con las acciones señaladas en el Plan Operativo. La elaboración de un nuevo formato denominado Evaluación General Trabajo de Grado, facilitara la obtención de información requerida por el CNA para garantizar a la sociedad que la Universidad cumple con los requisitos de calidad contemplados por ellos en busca de un proceso de acreditación para el programa, de igual manera será de gran ayuda para mantener un estadística acerca del impacto de los egresados al medio. El Plan Operativo para el Factor 9 del CNA permitirá optimizar la posición competitiva de la institución educacional en los diferentes campos en los que un egresado IPB-IB, puede llegar a desempeñarse con su perfil profesional.

\section{Referencias}

CAMPO, Álvaro; CHAPARRO, Fernando; CORREDOR, Martha; LAGO DE VERGARA, Diana; LONDOÑO, Guillermo; NIÑO, Jesús Virgilio y RIZO, Harold José. Sistema Nacional De Acreditación. Lineamientos para la Acreditación de Programas, Consejo Nacional de Acreditación.
Bogotá, D.C. Colombia. Noviembre de 2006.

ARISMENDY, Ana María; GONZALES, Ingrid Liliana y LÓPEZ, Nohora Milena. Evaluación de actividades académicas, investigativas y de proyección social desarrolladas por el programa de ingeniería biotecnológica como aporte a su proceso de autoevaluación con fines de acreditación. Cúcuta. Universidad Francisco de Paula Santander, 2011.

CONTRERAS, Leidy Katherine y COTE, Deisy. Estudio sobre la situación y tendencia de la ingeniería biotecnológica y las necesidades del sector productivo en el ámbito binacional. Cúcuta. Universidad Francisco de Paula Santander, 2011.

Informe De Condiciones De Calidad Para La Renovación Del Registro Calificado Del Programa Académico Ingeniería Biotecnológica. San José de Cúcuta, 2012.

Proyecto Educativo Institucional (PEI). Universidad Francisco de Paula Santander. Consejo Académico. San José de Cúcuta, 2007.

Proyecto Educativo De La Facultad (PEF). Universidad Francisco de Paula Santander. Consejo de Facultad Ciencias Agrarias y del Ambiente. San José de Cúcuta, 2008.

Proyecto Educativo Del Programa (PEP). Universidad Francisco de Paula Santander. Ingeniería Biotecnológica. Comité Curricular de Ingeniería Producción Biotecnológica. Ingeniería Biotecnológica. San José de Cúcuta, 2009. 